OPEN ACCESS

Edited by:

Liam Chen,

Johns Hopkins University,

United States

Reviewed by:

Jaleh Fallah,

Cleveland Clinic, United States

Rintaro Hashizume,

Northwestern University, United States

${ }^{*}$ Correspondence:

Milo Frattini

milo.frattini@ti.ch

Specialty section:

This article was submitted to

Neuro-Oncology and Neurosurgical

Oncology,

a section of the journal

Frontiers in Oncology

Received: 08 October 2019 Accepted: 27 December 2019

Published: 24 January 2020

Citation:

Marchi F, Sahnane N, Cerutti R,

Cipriani D, Barizzi J, Stefanini FM,

Epistolio S, Cerati M, Balbi S, Mazzucchelli L, Sessa F, Pesce GA, Reinert $M$ and Frattini $M$ (2020) The Impact of Surgery in IDH 1 Wild Type

Glioblastoma in Relation With the MGMT Deregulation.

Front. Oncol. 9:1569.

doi: 10.3389/fonc.2019.01569

\section{The Impact of Surgery in IDH 1 Wild Type Glioblastoma in Relation With the MGMT Deregulation}

\author{
Francesco Marchi ${ }^{1}$, Nora Sahnane ${ }^{2}$, Roberta Cerutti ${ }^{2}$, Debora Cipriani ${ }^{1}$, Jessica Barizzi ${ }^{3}$, \\ Federico Mattia Stefanini ${ }^{4}$, Samantha Epistolio ${ }^{3}$, Michele Cerati ${ }^{2}$, Sergio Balbi ${ }^{5}$, \\ Luca Mazzucchelli ${ }^{3}$, Fausto Sessa ${ }^{2}$, Gianfranco Angelo Pesce ${ }^{6}$, Michael Reinert ${ }^{1,7}$ and \\ Milo Frattini ${ }^{3 *}$
}

${ }^{1}$ Service of Neurosurgery, Neurocenter of the Southern Switzerland, Regional Hospital of Lugano, Lugano, Switzerland, ${ }^{2}$ Unit of Pathology, Department of Medicine and Surgery, University of Insubria-ASST Sette Laghi, Varese, Italy, ${ }^{3}$ Institute of Pathology, Locarno, Switzerland, ${ }^{4}$ Department of Statistics, Computer Science, Applications, University of Florence, Florence, Italy, ${ }^{5}$ Division of Neurological Surgery, Department of Biotechnology and Life Sciences, University of Insubria-ASST Sette Laghi, Varese, Italy, ${ }^{6}$ Radiation Oncology, Oncology Institute of Southern Switzerland, Bellinzona, Switzerland, ${ }^{7}$ Faculty of Medicine, University of Bern, Bern, Switzerland

Object: The treatment of choice in glioblastoma (GBM) is the maximal surgical extent of resection (EOR) followed by adjuvant chemo-radiotherapy. Furthermore, methylguanine-DNA methyltransferase (MGMT) promoter methylation is associated with prolonged overall survival (OS) and progression free survival (PFS). The objective of the present study is correlate the biomolecular aspects in relation with EOR.

Materials and methods: We analyzed a series of 116 patients with IDH-1 wild type GBM and different EOR (Gross Total Resection-GTR-, Partial Resection-PR- and Biopsy), treated with adjuvant chemo-radiotherapy. The MGMT status was analyzed in terms of promoter methylation and protein expression.

Results: When GTR was possible, OS and PFS were significantly better compared to the other two groups ( $p=0.001$ and $p=0.035$, respectively). MGMT methylation was significantly associated with better OS in the biopsy group $(p=0.022)$ and better $O S$ and PFS in PR ( $p=0.02$ and $p=0.012$, respectively), but not in the GTR group $(p=0.252$ for OS, $p=0.256$ for PFS) nor the PFS in the biopsy group ( $p=0.259)$. MGMT protein expression levels do not show any association with OS and PFS, regardless of the type of surgery.

Conclusions: Our study confirms the positive association of a safe maximal EOR with better OS and PFS, and indicates a positive prognostic value of MGMT methylation status only in case of the presence of residual tumor tissue. MGMT protein expression seems not to play a clinical role in relation with the type of surgery.

Keywords: glioblastoma, extent of resection, MGMT, overall survival, progression free survival, temozolomide 


\section{INTRODUCTION}

Glioblastoma (GBM) is the most common primary malignant brain tumor in adults (1). Currently, safe optimal surgical resection followed by adjuvant radiotherapy and chemotherapy is considered as the standard treatment approach for patients with GBM (2-4). However, despite advances in the last three decades and aggressive multimodal treatment, outcome remains poor for patients with GBM, with a median overall survival of 14-17 months from time at diagnosis $(2,3)$. Many studies have reported a positive correlation between the extent of resection (EOR) and the overall survival (OS) in patients with GBM, in particular for patients undergoing Gross Total Resection (GTR) with respect to whom receiving only a Subtotal Tumor Resection (STR) (5).

When GTR is not possible (due to several causes such as disease location and extension, general conditions of the patient), no clearly recognized criteria are proposed in the literature in order to stratify the STR group and, as a consequence, the threshold of EOR required for better prognosis remains controversial. Moreover, a recent observational retrospective study (6) has enrolled 38 patients who underwent PR and 78 biopsies and has pointed out that PR failed to improve OS and PFS compared with biopsy in patients with GBM ( $p=0.84$ and 0.48 , respectively). Even the propensity score matching (PSM) between the PR and biopsy groups, according with this study, did not show any significant difference in OS and PFS between the groups ( $p=0.51$ and 0.75 , respectively). The hazard ratios for OS and PFS of PR compared with biopsy were 0.98 and 0.73 , respectively; however, the difference was not statistically significant ( $p=0.96$ and 0.39 , respectively). Moreover, the surgical complication rate was higher in the PR group (14/32, $43.7 \%)$ than in the biopsy group $(9 / 78,11.5 \%)(p<0.01)$. The cited study confirms that no significant association and benefit has been clearly yet demonstrated between the different degrees of PR and the biomolecular markers in regards of OS and PFS.

Methylguanine-DNA methyltransferase (MGMT) plays the pivotal role in the management of GBM patients: hypermethylation of MGMT promoter (causing absence of MGMT protein expression) leads to a higher response to temozolomide (TMZ), thus improving the patients' outcome $(2,7,8)$. Furthermore, it has been discovered that additional mechanisms may decrease the MGMT expression. Approximately $20 \%$ of all patients with unmethylated GBM experiences an unexpected favorable outcome after chemoradiation, because mRNA expression was found to be unexpectedly low (9-11).

Only a few studies have investigated the influence of surgery on the clinical outcome in regards of the molecular markers $(4,12)$. Gessler et al. in their recent publication confirm that GTR is able to prolong PFS and OS when compared to incomplete

Abbreviations: EOR, Extent of Resection; GBM, Glioblastoma; GTR, Gross Total Resection; IDH, Isocitrate Dehydrogenase; IHC, immunohistochemistry; MGMT, methylguanine-DNA methyltransferase; PFS, Progression Free Survival; PR, Partial Resection; OS, Overall Survival; STR, Subtotal Resection; TMZ, temozolomide. resection, and the presence of methylation is a prognostic factor increasing significantly PFS and OS (4).

The aim of this study is to assess the relation between EOR and MGMT status (in terms of MGMT deregulation methylation and protein expression) by analyzing the clinical outcome (PFS and OS) of radio-chemotherapy treated IDH1 wild type GBM patients, in correlation with the type of surgery.

\section{MATERIALS AND METHODS}

This bi-center retrospective cohort study included patients with newly diagnosed histologically reviewed GBM with IDH-1 wild type status from 2004 until 2013.

This work has been conducted in compliance with the protocol, the current version of the Declaration of Helsinki, the ICH-GCP or ISO EN 14155 (as far as applicable) as well as all national legal and regulatory requirements. Data and samples have been collected and analyzed for the study purpose only after the required authorizations from the competent Ethics Committees were obtained (Rif. CE 3086-2016-01108).

Inclusion criteria consist of age $>18$ years, histological diagnosis of IDH-1 wild type GBM (WHO IV), therapy with TMZ according with the Stupp scheme (60 Gray radiotherapy and concomitant chemotherapy with TMZ, followed by six cycles of maintenance TMZ), death caused by GBM, tissue availability for biomolecular analyses.

The OS (defined as the time from surgery to the date of death) and PFS (defined as the time from the first radio-chemotherapy treatment to the date of clinical or radiological progression according with the RANO criteria) were analyzed. Regarding the type of surgery, three groups were defined according with the post-op MRI performed in the first $72 \mathrm{~h}$ : GTR (with no contrast-enhancing residual tissue visible on T1 injected MRI sequences), incomplete Partial Resection (PR) (with evidence of contrast-enhancing residual tumor) and Biopsy.

\section{Molecular Analyses MGMT Promoter Methylation}

Tissues for genomic DNA isolation were dissected manually from three $8-\mu \mathrm{m}$ sections and DNA was obtained using automatic extraction (Maxwell, Promega, Madison, WI, USA). About 50-100 ng of DNA were subjected to bisulphite treatment using EZ DNA Methylation-Gold TM kit (Zymo Research, Irvine, CA, USA). Methylation status of six consecutive cytosines of MGMT promoter (chr10:131,265,507-131,265,556) was assessed by PCR-pyrosequencing of bisulphite-treated DNA by using MGMT Plus kit according to the recommended protocol (Diatech Pharmacogenetics, Jesi, Italy). A cut-off of $10 \%$ was set to score presence of promoter methylation. This value was determined calculating the limit of negative controls (DNA samples from 15 FFPE healthy brain tissues) for each cytosine (mean of methylation ratio adding $2 \times$ the Standard Deviation) assuming a Gaussian distribution of the raw signal from negative samples. The limit corresponded to $95 \%$ of the observed negative values. 


\section{MGMT Immunohistochemistry}

Immunohistochemical reactions for MGMT protein were performed on whole tissue sections obtained from formalinfixed, paraffin-embedded (FFPE) tumor blocks. Three - $\mu$ m-thick sections were deparaffinized, rehydrated and pretreated with citrate buffer $\mathrm{pH} 6$ in microwave oven for $20 \mathrm{~min}$. Monoclonal primary antibody anti-MGMT, clone MT3.1 (Chemicon International, Temecula, CA, USA) was used at a dilution of $1 / 400$ and applied overnight at $4^{\circ} \mathrm{C}$, followed by a polymeric detection system (Ultravision DAB Detection System, LabVision, Fremont, CA, USA) according to the manufacturer's protocol. According to the literature, immunohistochemical positivity was scored when more than $5 \%$ of neoplastic cells showed an intense nuclear staining $(13,14)$.

\section{Statistical Analyses}

Mean and median values were calculated at first to summarize results of each variable. The relative chi-square values were calculated on pairs of variables to describe the statistical association existing among variables: null hypothesis stating the lack of marginal association between pairs of variables (after discretization) was assessed through the chi-square test.

OS and PFS curves for censored data were obtained using the Kaplan-Meier estimator; comparisons of curves given different molecular characterizations were performed by logrank tests. PFS curves were also estimated and tested within strata defined by the variable of surgery.

All the analyses, graphs and reports were performed using the $\mathrm{R}$ software $[\mathrm{R}]$ and the following $\mathrm{R}$ packages: survival, bootstrap, rmarkdown, knitr (15-18).

\section{RESULTS}

The study includes 116 patients, 57 females (49.1\%) and 59 males (50.9\%). Among them, 81 underwent GTR of the tumor (69.8\%), 18 PR (15.5\%) while in 17 cases only biopsy was performed (14.7\%). In 92 patients (corresponding to $79.3 \%$ of the whole cohort), we observed progression of the disease (PD), while the remaining 24 cases (20.7\%) include both the six patients who are still alive $(N=6)$ and the 18 patients who deceased for other causes with no evidence of tumor progression.

Concerning the biomolecular aspects, 71 samples showed absence of MGMT promoter methylation tumors (61.2\%), 41 methylation $(35.3 \%)$ while in four samples the methylation status was not evaluable (3.4\%). The immunohistochemical evaluation of the MGMT protein revealed a positive expression in 54 samples (46.5\%) and a negative expression in 44 cases (37.9\%), while in 18 cases (15.5\%) the assay did not give evaluable results (Table 1). As for clinical data, OS was 15.5 months and PFS was 7 months.

We then analyzed the correlation between the type of surgery and the OS (Figures 1A,B). Patients who underwent a GTR had a significantly better OS (17 months) compared with those in whom a PR (14 months) or a biopsy ( 9 months) had been performed (Log-rank $p=0.001$; GTR: $\mathrm{HR}=0.3521$, 95\%CI: 0.1989, 0.6235; PR: HR = 0.3926, 95\%CI: 0.1908, 0.8075) (Figure 1A). Analogously, grouping the patients who sustained
TABLE 1 | Synoptic overview of the patient population.

\begin{tabular}{|c|c|c|}
\hline Patients & 116 & \\
\hline IDH1 wild type GBM WHO IV & 116 & \\
\hline Female & 57 & $49.1 \%$ \\
\hline Male & 59 & $50.9 \%$ \\
\hline GTR & 81 & $69.8 \%$ \\
\hline PR & 18 & $15.5 \%$ \\
\hline Biopsy & 17 & $14.7 \%$ \\
\hline Surgery + Radio-chemotherapy & 116 & \\
\hline Deceased & 110 & $94.8 \%$ \\
\hline Alive & 6 & $5.1 \%$ \\
\hline Progression Disease (PD) & 92 & $79.3 \%$ \\
\hline Alive with no evidence of PD & 6 & $5.1 \%$ \\
\hline Deceased with no evidence of PD & 18 & $15.5 \%$ \\
\hline MGMT methylated & 41 & $35.3 \%$ \\
\hline MGMT non-methylated & 71 & $61.2 \%$ \\
\hline MGMT non definable & 4 & $3.4 \%$ \\
\hline MGMT protein expression positive & 54 & $46.5 \%$ \\
\hline MGMT protein expression negative & 44 & $37.9 \%$ \\
\hline MGMT expression not evaluable & 18 & $15.5 \%$ \\
\hline
\end{tabular}

GBM, Glioblastoma; GTR, Gross Total Resection; MGMT, methylguanine-DNA methyltransferase; PR, Partial Resection.

PR and biopsy, we confirmed a significant longer OS for patients subjected to GTR (GTR = 17 months, PR + Biopsy $=11.5$ months, Log-rank $p=0.0333$; GTR: $\mathrm{HR}=1.571,95 \% \mathrm{CI}: 1.0316$, 2.3923) (Figure 1B). Similar results were obtained by comparing the type of surgery and PFS (Figures 1C,D). Indeed, the GTR group presented a longer PFS compared with the PR group and the biopsy group (GTR $=8.25$ months, $P R=7.50$ months, Biopsy $=4.00$ months, Log-rank $p=0.0352$; GTR: $\mathrm{HR}=0.3681$, 95\%CI: 0.1738, 0.7799; PR: HR = 0.4444, 95\%CI: 0.1877, 1.0522)

(Figure 1C). On the contrary, GTR did not give a significant greater PFS compared with the value of the other two groups considered together $(\mathrm{GTR}=8.25$ months, $\mathrm{PR}+$ Biopsy $=7.00$, Log-rank $p=0.1187$; PR + biopsy: HR $=1.4774,95 \% \mathrm{CI}: 0.9211$, 2.3698) (Figure 1D).

Afterwards, the OS and the PFS were analyzed in relation with the MGMT promoter methylation and the protein expression evaluated by immunohistochemistry (IHC) (Figures 2A-D). The OS is significantly better in MGMT methylated GBMs than in MGMT unmethylathed ones (methylated: 19.5 months, unmethylathed: 14 months, Log-rank $p=0.0056$; U: HR $=1.7653$, 95\%CI: 1.174, 2.6544) (Figure 2A). Same positive correlation, statistically significant, was found for PFS, with 9 months before progression in MGMT methylated patients and 7 months for MGMT unmethylated ones (Log-rank $p=0.0347$; $\mathrm{U}: \mathrm{HR}=1.6014,95 \% \mathrm{CI}: 1.037,2.473$ ) (Figure 2B).

Regarding the levels of MGMT protein expression, patients who had low MGMT protein expression had a significantly improved OS compared with patients who had high MGMT protein expression (18 vs. 13 months; Log-rank $p=0.0148$; Pos: $\mathrm{HR}=1.6929,95 \% \mathrm{CI}: 1.1165,2.567$ ) (Figure 2C). On the contrary, no significant correlation was observed for MGMT 


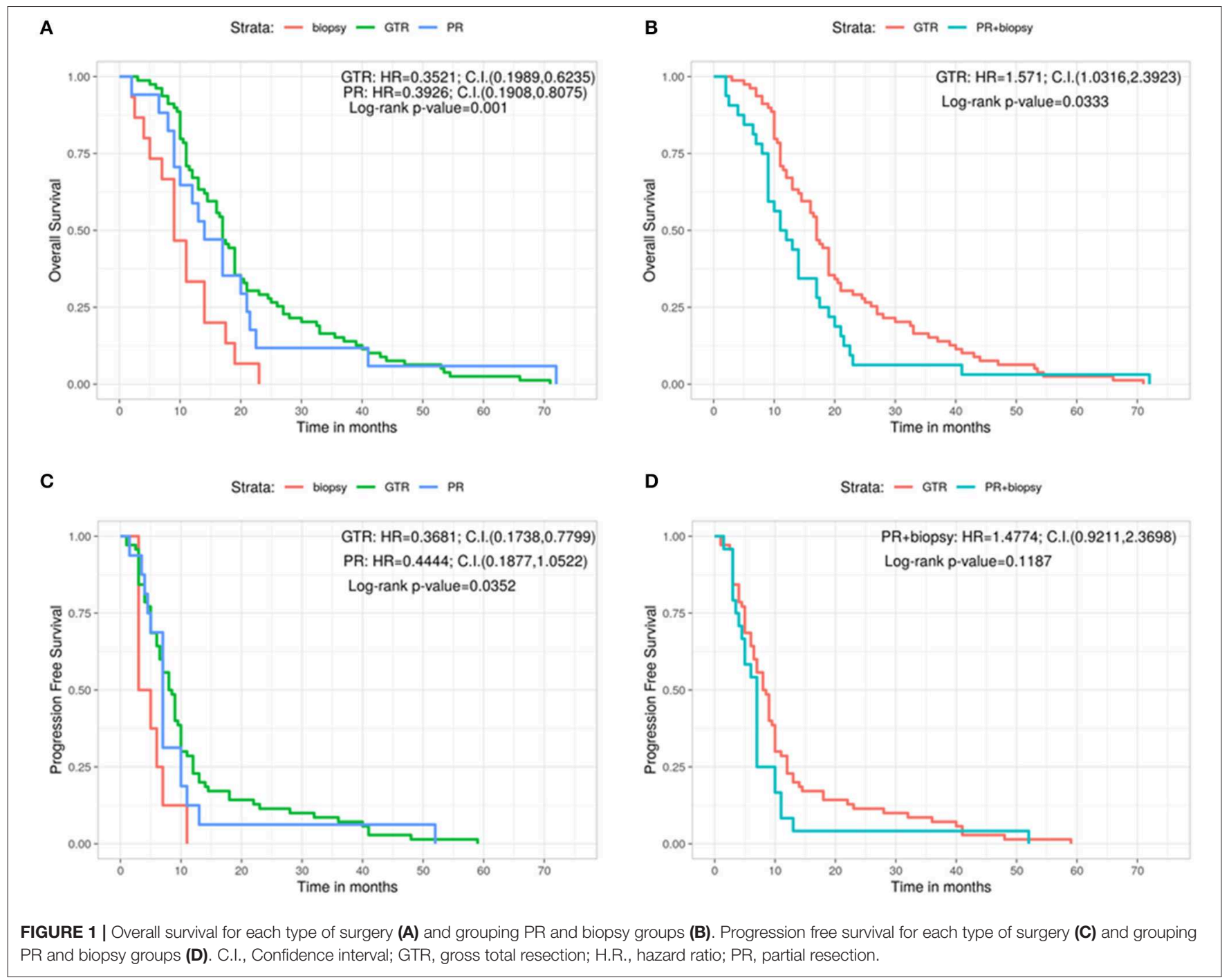

protein expression in regards of PFS (8.75 months for GBMs showing low protein expression and 7 months for those with high protein expression, Log-rank $p=0.3166$; Pos: $\mathrm{HR}=1.2486$, 95\%CI: 0.8013, 1.9458) (Figure 2D).

Furthermore, we analyzed the outcome (in terms of both OS and PFS) subdividing the cohort on the basis of the three different types of surgery, in relation with the methylation status of the MGMT gene (Figures 3A-F). No significant correlation was found in patients with GTR between OS and methylation status (methylated $=19$ months, unmethylated $=16$ months, Log-rank $p=0.252 ; \mathrm{U}: \mathrm{HR}=1.3125,95 \% \mathrm{CI}$ : 0.8277, 2.0813) (Figure 3A). A positive correlation was shown, instead, in the PR group, in which MGMT methylated patients had a better OS compared with the unmethylated ones (methylated $=31.8$ months, unmethylated $=13.0$ months, Log-rank $p=0.0205$; $\mathrm{U}: \mathrm{HR}=8.5176,95 \% \mathrm{CI}: 1.0472,69.2787$ ) (Figure 3B). The same positive statistically significant correlation was observed in patients who underwent biopsy (methylated $=21$ months, unmethylated $=9$ months, Log-rank $p=0.0226$; $\mathrm{U}: \mathrm{HR}=$ undefined, 95\%CI: 0, $\infty$ ) (Figure 3C).
As regards the PFS, patients who underwent a GTR and were MGMT methylated did not show a better outcome if compared with patients carrying MGMT unmethylated GBM (methylated $=9$ months, unmethylated $=7$ months, Log-rank $p=0.256$; $\mathrm{U}: \mathrm{HR}=1.3215,95 \% \mathrm{CI}: 0.8134,2.147$ ) (Figure 3D). On the contrary, a statistically significant better PFS was noted in MGMT methylated patient with respect to MGMT unmethylated ones in the PR group (methylated $=13$ months, unmethylated $=7$ months, Log-rank $p=0.0117$; U: HR $=9.0791$, 95\%CI: 1.141, 72.2475) (Figure 3E). Finally, absence of correlation between PFS and the methylation status was observed in the group of patients who underwent a biopsy (methylated $=5$ months, unmethylated $=3$ months, Log-rank $p=0.2982$; U: $\mathrm{HR}=2.5982$, 95\%CI: $0.2975,22.6896)$ (Figure 3F).

Moreover, we analyzed the same variables (clinical outcome and EOR) on the light of the results of MGMT protein expression (Figures 4A-F). In terms of OS, patients who underwent a GTR with a low protein expression had a significant better outcome with respect to patients with high MGMT expression (low protein expression $=19.8$ months, high protein 

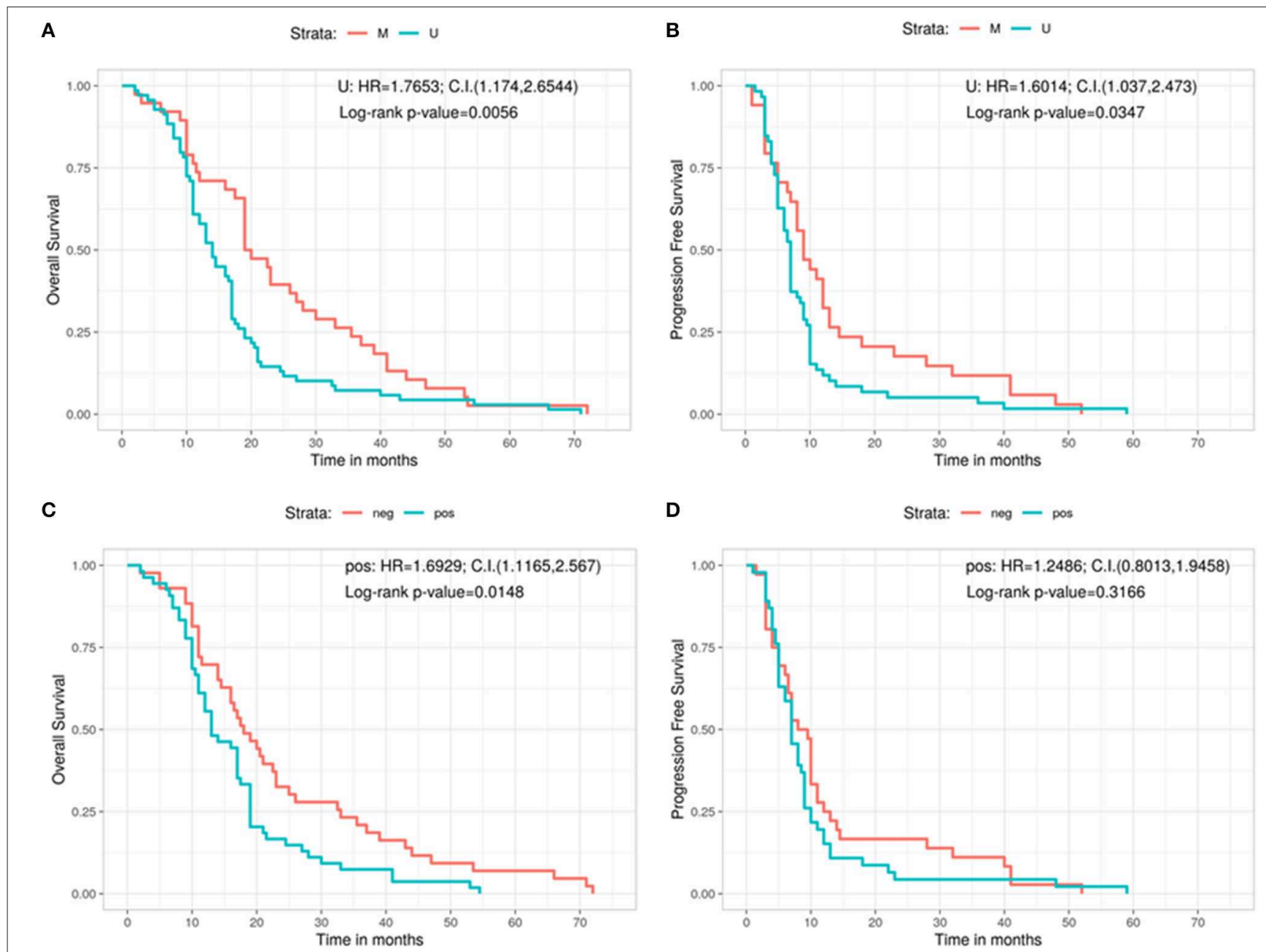

FIGURE 2 | Overall survival (A) and progression free survival (B) according with the MGMT methylation status. Overall survival (C) and progression free survival (D) according with the immunohistochemistry results. C.I., Confidence interval; H.R., hazard ratio; M, methylated; neg, IHC negative; pos, IHC positive; U, unmethylated.

expression $=16.5$ months, Log-rank $p=0.0476$; Pos: $\mathrm{HR}=$ 1.6592, 95\%CI: 1.0144, 2.714) (Figure 4A). On the contrary, no significant correlations were found in the PR and in the biopsy groups regarding MGMT protein expression in terms of OS: 17 months for low protein expression patients vs. 12.5 months for those with high protein expression in the PR group (Log-rank $p$ $=0.3702$; Pos: $\mathrm{HR}=1.6552$, 95\%CI: 0.5554, 4.9325) (Figure 4B), and 11 months in low protein expression patients vs. 8 months in patients with high MGMT expression in the biopsy group (Log-rank $p=0.42$; Pos: $\mathrm{HR}=1.596,95 \% \mathrm{CI}$ : 0.4772, 5.3374) (Figure 4C).

As regards PFS, IHC showed no significant relation between low and high protein expression patients in any group. In the GTR patients, the difference in PFS between low expressed and high expressed cases was 9.5 vs. 8 months $(\log -\operatorname{rank} p=0.6387$; Pos: $\mathrm{HR}=1.1313,95 \% \mathrm{CI}: 0.6718,1.905$ ) (Figure 4D). In the PR group, PFS was 10 months for low protein expression patients vs. 7 months for patients with high MGMT protein levels (Logrank $p=0.3034$; Pos: $\mathrm{HR}=1.8299$, 95\%CI: 0.5637, 5.9407)
(Figure 4E). In the biopsy group, patients showing low protein expression level had a PFS of 5 months compared with 3 months of those with a high MGMT protein expression (Log-rank $p=$ 0.259; Pos: $\mathrm{HR}=2.3493$, 95\%CI: 0.3908, 14.1236) (Figure 4F).

\section{DISCUSSION}

Our paper presents a bicentric, retrospective study including a series of patients affected by IDH-1 wild type GBM treated with chemotherapy and radiotherapy after surgery.

Firstly, compared to the data in the literature, we tried to define three new unambiguous categories of surgical treatment: GTR, when no evidence of residual tumor on the T1 injected post-op sequences MRI; PR, if any enhancement is visible (independently of the residual volume); and biopsy, if only a small piece of tumor is taken for analysis. We think that this categorization is a novelty in literature considering the $\mathrm{PR}$ as the presence of residual tumor, regardless its volume. All the other 


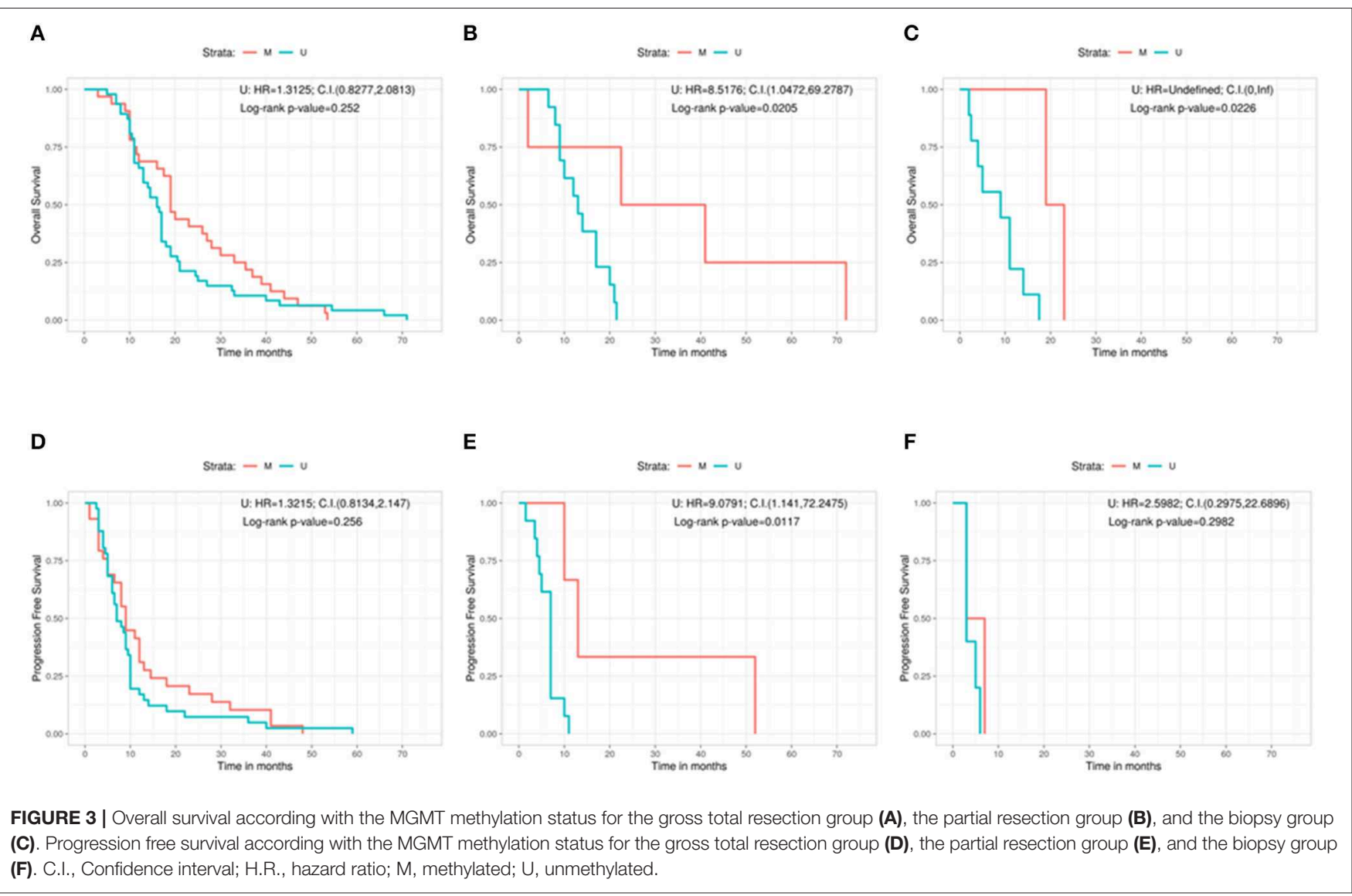

studies, in fact, have defined the residual volume in a percentage that, in the majority of cases, may be subjective.

Our results confirm the well established statement for which, when feasible, GTR is the gold standard to achieve in the surgical treatment of GBMs with a longer term OS in this group of patients vs. both the PR group and the biopsy group (17 vs. 14 vs. 9 months for the different groups, respectively). Similar data are obtained comparing the GTR group with the PR + biopsy combined one (17 vs. 11.5, respectively). However, as stated above, the GTR in our work is the complete absence of residual tumor on the postop MRI and not, as in literature, the variable majority of tumor resected ( $>95$ or $>97 \%$ ) considering the difficulty in the objective calculation of the percentage of the remnant tumor.

Regarding the PFS, we observed the same significant positive relation in favor of the GTR group compared with the PR and biopsy groups taken singularly ( 8.25 vs. 7.5 vs. 4 , respectively) but not when the latter two ( $\mathrm{PR}+$ biopsy) are assembled together. Also these data confirm the already published ones but, differently, in our work, the PR is considered any enhancement of any size visible on the postoperative MRI.

In respect of the MGMT methylated status and the better outcome, our results are in line with the main series published in literature $(7,19)$ with a median OS of 19.5 months for patients with a MGMT methylated GBM vs. 14 months for patients with an MGMT unmethylated GBM and 2 months more of PFS between the two groups. However, while the better prognosis in terms of OS was confirmed by protein expression levels assessed by IHC, no significant correlation between the two groups (low and high MGMT protein expression) was shown for the PFS with this analysis. Therefore, our study confirms the lower diagnostic value of IHC as compared to the evaluation of the MGMT methylation status.

The most interesting results have been obtained matching the MGMT status and the EOR. While we observed that the MGMT status is positively and significantly correlated to the clinical outcome in the PR group (OS of 31.8 months for patients with MGMT methylated GBM with respect to only 13 months for MGMT unmethylated GBM, PFS of 13 vs. 7 months, respectively), in the group of patients who underwent a GTR we did not observe any significant association between OS or PFS and the methylation status of MGMT gene. Likewise, the simple biopsy did not change significantly the outcome in terms of PFS in methylated vs. unmethylated patients with only 2 months achieved before disease progression. However, in terms of OS, patients characterized by an MGMT methylated GBM have an advantage of 12 months with respect to patients with an MGMT unmethylated tumor. Our results confirm those recently published but using clearly, objective and widely applicable categories of $\operatorname{EOR}(4,12)$. Therefore, we can postulate that the identification of MGMT promoter methylation may identify a group of GBM patients who are correlated with a better 


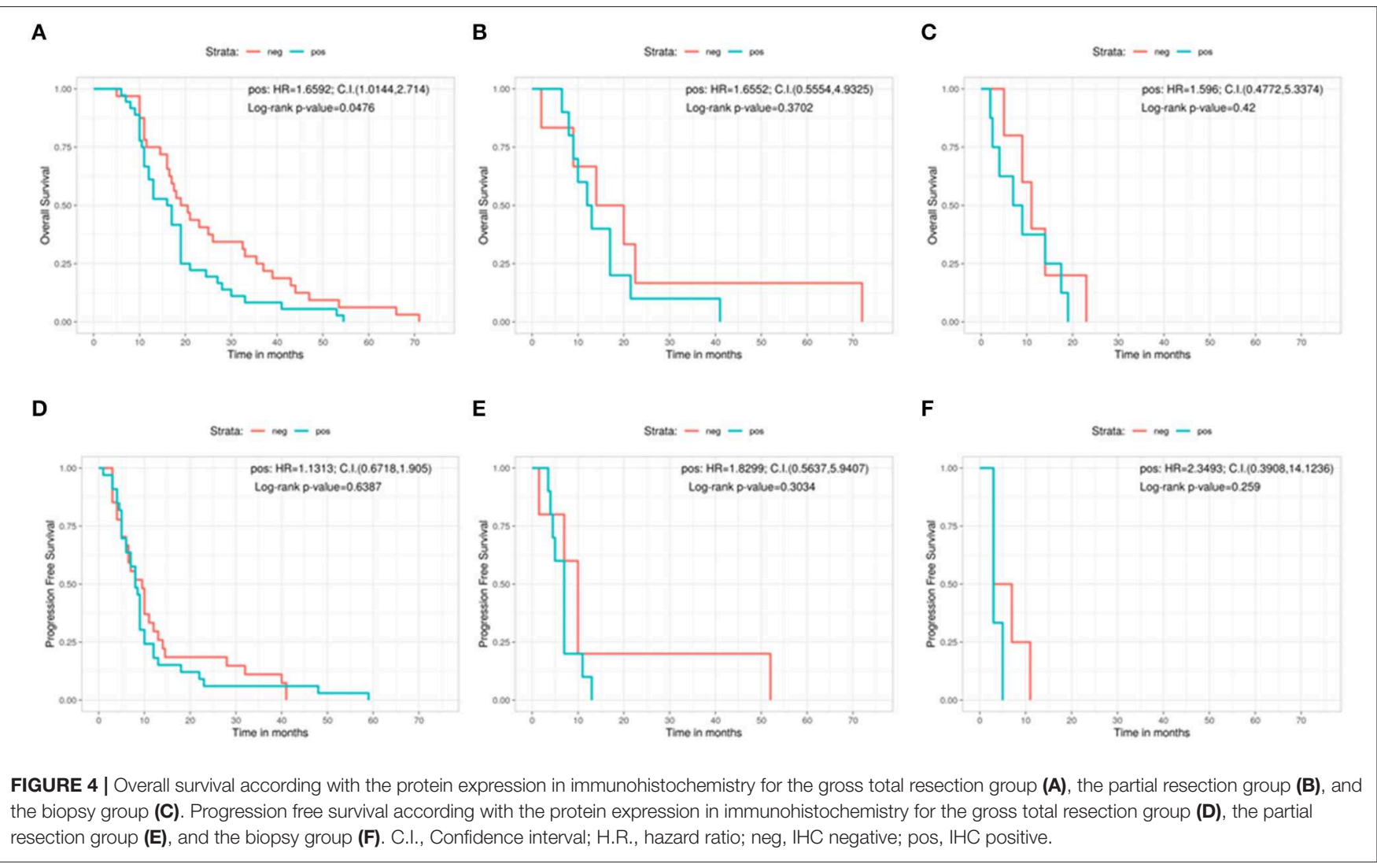

response to the combined chemo-radiotherapy treatment only if the neoplastic tissue is still present. On the contrary, patients who are bona fide radically resected, will experience the same follow-up with respect to the combined chemo-radiation therapy.

IHC represents the classic worldwide used method for the detection of protein expression. However, especially for MGMT protein (as for other proteins located in cell nucleus), it can be sometimes hard to be evaluated, leading to the conclusion that MGMT promoter methylation should be the preferred method for assessing MGMT deregulation instead of IHC. In fact, literature reports that sometimes there is no correlation between MGMT expression and its promoter methylation (20). Also in our series IHC does not confirm the expected significant correlation between low protein expression and better clinical outcome in PR and biopsy groups. Indeed, the only favorable significant correlation (borderline, $p=0.047$ ) for the low protein expression was noted in the OS for the patients who underwent GTR: they presented almost 20 months of survival vs. 16.5 months of those with samples expressing high levels of MGMT protein. Neither the GTR nor the PR or the biopsy group showed a significant relation between IHC and PFS.

Beside IHC limits, also the definition of MGMT promoter methylation is sometimes challenging, however literature reports some cut-off values that can be used to define a sample as positive for MGMT methylation (21). In our work we applied a cutoff of $10 \%$ that was decided on the bases of an internal control evaluation of a cohort of negative (healthy tissues) samples.
Our study present some limitations, such as the number of patients included in the analysis is small if compared to other series, even if not inferior to the majority of studies published. For this reason, the results of the present work are in line with those in the literature and seem to be not innovative. However, several aspects are not exhaustively treated in literature and some features can be helpful in everyday practice as, for example, the simple classification between GTR and PR or the importance of radio-chemotherapy in case of residual enhancement on the postoperative images.

To conclude, the present study confirms the better outcome in patients with GBM who sustained a GTR: maximal EOR in surgery seems to be confirmed as the most important prognostic value for OS and PFS in the treatment of GBM patients, thus indicating that, whenever possible, this is the goal that must be pursued by clinicians. Under these conditions, the most relevant biomarker, MGMT, does not seem to play any prognostic role. Theoretically and provocatively, the present study states that chemo-radiotherapy, in presence of complete resection, could not influence significantly OS and PFS, playing a substantial role only in case of residual tumor. When surgery is not possible, the MGMT methylated status is proven to be a favorable marker for OS and PFS in patients with remnant tumor after an incomplete tumor resection (in both PR and biopsied patients). On the contrary, IHC expression does not correlate with different OS or PFS in relation with the type of surgery, thus confirming the discrepancy between protein expression and MGMT methylation 
status evaluation and suggesting a superior predictive role of the latter.

We think that the current study, to the best of our knowledge, is one of the few studies which correlates biological aspects and different type of surgery in GBM patients treated with a combined chemo-radiotherapy. However, considering the continuous changes in the field of brain tumors, further studies are needed in order to confirm our data and to identify other possible correlations between the newest biological markers, the clinical outcome and the surgical treatment in patients with GBM.

\section{DATA AVAILABILITY STATEMENT}

The raw data supporting the conclusions of this article will be made available by the authors, without undue reservation, to any qualified researcher.

\section{ETHICS STATEMENT}

This work has been conducted in compliance with the protocol, the current version of the Declaration of Helsinki, the ICH-GCP

\section{REFERENCES}

1. Louis DN, Perry A, Reifenberger G, Von Deimling A, FigarellaBranger D, Cavenee WK, et al. The 2016 World Health Organization classification of tumors of the central nervous system: a summary. Acta Neuropathol. (2016) 131:803-20. doi: 10.1007/s00401-016-1 545-1

2. Stupp R, Mason WP, van den Bent MJ, Weller M, Fisher B, Taphoorn $\mathrm{MJB}$, et al. Radiotherapy plus concomitant and adjuvant temozolomide for glioblastoma. N Engl J Med. (2005) 352:987-96. doi: 10.1056/NEJMoa0 43330

3. Weller M, van den Bent M, Hopkins K, Tonn JC, Stupp R, Falini $\mathrm{A}$, et al. EANO guideline for the diagnosis and treatment of anaplastic gliomas and glioblastoma. Lancet Oncol. (2014) 15:e395-403. doi: 10.1016/S1470-2045(14)70011-7

4. Gessler F, Bernstock JD, Braczynski A, Lescher S, Baumgarten P, Harter PN, et al. Surgery for glioblastoma in light of molecular markers: impact of resection and MGMT promoter methylation in newly diagnosed IDH-1 wildtype glioblastomas. Neurosurgery. (2018) 84:190-7. doi: 10.1093/neuros/n yy049

5. Brown TJ, Brennan MC, Li M, Church EW, Brandmeir NJ, Rakszawski KL, et al. Association of the extent of resection with survival in glioblastoma: a systematic review and metaanalysis. JAMA Oncol. (2016) 2:1460-9. doi: 10.1001/jamaoncol.201 6.1373

6. Byun J, Kim YH, Nam SJ, Park JE, Cho YH, Kim HS, et al. Comparison of survival outcomes between partial resection and biopsy for primary glioblastoma: a propensity score-matched study. World Neurosurg. (2019) 121:e858-66. doi: 10.1016/j.wneu.2018.0 9.237

7. Hegi ME, Diserens AC, Gorlia T, Hamou MF, de Tribolet N, Weller $\mathrm{M}$, et al. MGMT gene silencing and benefit from temozolomide in glioblastoma. N Engl J Med. (2005) 352:997-1003. doi: 10.1056/NEJMoa04 3331

8. Esteller M, Hamilton SR, Burger PC, Baylin SB, Herman JG. Inactivation of the DNA repair gene O6-methylguanine-DNA methyltransferase by promoter hypermethylation is a common event in primary human neoplasia. Cancer Res. (1999) 59:793-7. or ISO EN 14155 (as far as applicable) as well as all national legal and regulatory requirements. Data and samples have been collected and analyzed for the study purpose only after the required authorizations from the competent Ethics Committees were obtained (Rif. CE 3086-2016-01108).

\section{AUTHOR CONTRIBUTIONS}

FM and NS collected all clinical data and wrote the first draft of the manuscript. FM, DC, FS, GP, MR, and MF selected the cohort for the analyses. JB, MC, LM, FS, and GP selected all the specimens at histologic level. NS, RC, SE, and SB performed all the molecular characterization. SE, RC, and MF evaluated MGMT results. JB, MC, LM, FS, and GP evaluated IHC experiments. FMS was responsible for all the statistical analyses. FM, NS, and SE prepared the final version of the manuscript. FM, FS, GP, MR, and MF supervised the whole project.

\section{FUNDING}

This study was supported by Fondazione Ticinese contro il Cancro.

9. Everhard S, Tost J, El Abdalaoui H, Crinière E, Busato F, Marie Y, et al. Identification of regions correlating MGMT promoter methylation and gene expression in glioblastomas. Neuro Oncol. (2009) 11:348-56. doi: 10.1215/15228517-2009-001

10. Ramakrishnan V, Kushwaha D, Koay DC, Reddy H, Mao $\mathrm{Y}$, Zhou L, et al. Post-transcriptional regulation of $\mathrm{O}(6)$ methylguanine-DNA methyltransferase MGMT in glioblastomas. Cancer Biomark. (2011) 10:185-93. doi: 10.3233/CBM-201 2-0245

11. Kreth S, Limbeck E, Hinske LC, Schütz SV, Thon N, Hoefig K, et al. In human glioblastomas transcript elongation by alternative polyadenylation and miRNA targeting is a potent mechanism of MGMT silencing. Acta Neuropathol. (2013) 125:671-81. doi: 10.1007/s00401-013-1 081-1

12. Kreth FW, Thon N, Simon M, Westphal M, Schackert G, Nikkhah G, et al. Gross total but not incomplete resection of glioblastoma prolongs survival in the era of radiochemotherapy. Ann Oncol. (2013) 24:3117-23. doi: 10.1093/annonc/mdt388

13. Brell M, Tortosa A, Verger E, Gil JM, Viñolas N, Villá $\mathrm{S}$, et al. Prognostic significance of O6-methylguanine-DNA methyltransferase determined by promoter hypermethylation and immunohistochemical expression in anaplastic gliomas. Clin Cancer Res. (2005) 11:5167-74. doi: 10.1158/1078-0432.CCR-0 5-0230

14. Cao VT, Jung TY, Jung S, Jin SG, Moon KS, Kim IY, et al. The correlation and prognostic significance of MGMT promoter methylation and MGMT protein in glioblastomas. Neurosurgery. (2009) 65:866-75. doi: 10.1227/01.NEU.0000357325.90347.A1

15. Xie Y. A Comprehensive Tool for Reproducible Research in R. Implementing Reproducible Computational Research. New York, NY: CRC Press Group (2014).

16. Therneau T. A Package for Survival Analysis in S_. Version. (2015). Available online at: https://cran.r-project.org/package=survival (accessed December 11, 2018).

17. Allaire J. Dynamic Documents for R. R Package Version 1.11. (2018). Available online at: https://rmarkdown.rstudio.com/ (accessed December 11, 2018).

18. Team RC. A Language and Environment for Statistical Computing. (2018). Available online at: https://www.r-project.org/ (accessed December 11, 2018). 
19. Stupp R, van den Bent MJ, Hegi ME. Optimal role of temozolomide in the treatment of malignant gliomas. Curr Neurol Neurosci Rep. (2005) 5:198-206. doi: 10.1007/s11910-005-0047-7

20. Rodriguez FJ, Thibodeau SN, Jenkins RB, Schowalter KV, Caron BL, O'Neill BP, et al. MGMT immunohistochemical expression and promoter methylation in human glioblastoma. Appl Immunohistochem Mol Morphol. (2008) 16:59-65. doi: 10.1097/PAI.0b013e3180 $2 \mathrm{fac} 2 \mathrm{f}$

21. Brigliadori G, Foca F, Dall'Agata M, Rengucci C, Melegari E, Cerasoli, et al. Defining the cutoff value of MGMT gene promoter methylation and its predictive capacity in glioblastoma. J Neurooncol. (2016) 128:333-9. doi: 10.1007/s11060-016-2116-y
Conflict of Interest: The authors declare that the research was conducted in the absence of any commercial or financial relationships that could be construed as a potential conflict of interest.

Copyright (C) 2020 Marchi, Sahnane, Cerutti, Cipriani, Barizzi, Stefanini, Epistolio, Cerati, Balbi, Mazzucchelli, Sessa, Pesce, Reinert and Frattini. This is an open-access article distributed under the terms of the Creative Commons Attribution License (CC $B Y)$. The use, distribution or reproduction in other forums is permitted, provided the original author(s) and the copyright owner(s) are credited and that the original publication in this journal is cited, in accordance with accepted academic practice. No use, distribution or reproduction is permitted which does not comply with these terms. 